\title{
The Electromagnetic Unification of Four Forces and Electromagnetic Structures of Particles
}

\author{
Zi-Jian Cai \\ CaiFortune Consulting, Suzhou, China \\ Email:hrsh8@126.com
}

How to cite this paper: Cai, Z.-J. (2018) The Electromagnetic Unification of Four Forces and Electromagnetic Structures of Particles. Open Access Library Journal, 5: e4514.

https://doi.org/10.4236/oalib.1104514

Received: March 18, 2018

Accepted: April 15, 2018

Published: April 18, 2018

Copyright (๐ 2018 by author and Open Access Library Inc.

This work is licensed under the Creative Commons Attribution International License (CC BY 4.0).

http://creativecommons.org/licenses/by/4.0/

(c) (i) Open Access

\begin{abstract}
Recently, the book "Electromagnetic Unification of Four Forces" (ISBN978-3-659-76798-2) was published, compiling the progressions in physics made by the world people, manifested in television while led notably by me and the Members in the world satellite networks from India, Zhejiang and USA. Many important progressions were achieved, such as the spatially localized electromagnetic mass structure; the electromagnetic multi-hairy structure; the electromagnetic orienting force; the thermodynamic stabilization of strong force from electromagnetic multi-hairy hybrid; the weak processes from hybrid split and the electromagnetic oscillatory neutrino structure; the gravitational force as electromagnetic curving and shifting red from electromagnetic orienting force; and so on; electromagnetic unifying the four forces. Afterwards, it was also explained the quantum theory of Planck with the electromagnetic mass hairy structure because all photons emitted from electron possessed identical electrical hairy strength as electron with frequency to differentiate the energy only. Besides, from the various decay products, it was further proposed the electromagnetic structures of particles most important in physics, including those combined baryons based on linear proton and hexagonal neutron, such as the proton, neutron, $\Lambda 0$ baryon, $\Sigma$ baryons, and $\Xi$ baryons; those based on kaons from colliding pieces such as the kaons, $\eta$ baryons, charm quarks, and bottom quarks; those containing the $\mathrm{W} / \mathrm{Z}$ bosons with wavelength equivalent to hybrid interval, such as the $\mathrm{W} / \mathrm{Z} /$ Higgs bosons and top quarks. It is expected that this review can introduce all these important progressions to readers for academic propagation and citation.
\end{abstract}

\section{Subject Areas}

Theoretical Physics, Particle Physics, Nuclear Physics, Modern Physics 


\section{Keywords}

Electromagnetic Mass Structure, Electromagnetic Orienting Force, Electromagnetic Multi-Hairy Structure, Strong Force from Electromagnetic Hybrid and Thermodynamics, Electromagnetic Oscillatory Neutrino Structure, Linear Proton, Hexagonal Neutron

\section{Introduction}

Recently, the author published the book "Electromagnetic Unification of Four Forces" (ISBN978-3-659-76798-2) [1]. As the premise, the energy was present as the frequency of electromagnetic wave or frequency of light in atomic physics, therefore all forces with energy should be unified with the electromagnetic force. In the book, it was compiled the progressions in physics organized by the author Zi-Jian Cai with the India Member, Zhejiang Member, and US Members in the world satellite networks and accomplished altogether with the anonymous people in worldwide television from 2005 to 2009. During such process there was achieved many very important physics progressions, such as the non-relativity of ideal time and space, electromagnetic mass structure, electromagnetic multi-hairy structure, electromagnetic orienting force, oscillatory neutrino structure, and so on. There was explained and unified the strong force, weak force and gravitational force with the electromagnetic force, realizing the unification of four forces in physics. The television and satellites were the new public media announcing and propagating these achievements, widely influencing the people of the whole world, and vividly manifesting the unprecedented originality of the achievements.

Afterwards, the author Zi-Jian Cai also explained the quantum theory of Planck with the electromagnetic mass hairy structure. All photons emitted from electron possessed identical electrical hairy strength as electron, so that only the frequency differentiated the energy [2]. In addition, from the various decay products, the author Zi-Jian Cai further proposed the electromagnetic structures of particles most important in physics, while classified them into those combined baryons based on linear proton and hexagonal neutron, such as the proton, neutron, $\Lambda 0$ baryon, $\Sigma$ baryons, and $\Xi$ baryons; those based on kaons from colliding pieces such as the kaons, $\eta$ baryons, charm quarks, and bottom quarks; those containing the $\mathrm{W} / \mathrm{Z}$ bosons with wavelength equivalent to hybrid interval, such as the W/Z/Higgs bosons and top quarks [3].

For all such milestone progressions in physics as the electromagnetic unification of four forces and establishment of electromagnetic structures of particles, it is obviously worthy to review their contents briefly as the paper on Open Access journal for the convenience of academic propagation and citation. In this review article, it is attempted to undertake and finish this task. 


\section{Brief Review of Milestone Book: Electromagnetic Unification of Four Forces}

\subsection{The Non-Relativity of Ideal Time and Space}

In the book, it was demonstrated the non-relativity of ideal time and space in the first chapter [2] [4]. As proposal, the ideal reference system $\mathrm{O}$ was stationary, the ideal reference system $\mathrm{A}$ and $\mathrm{B}$ moved at constant velocity $\mathrm{v}$ and $-\mathrm{v}$ respectively. From the reference system $\mathrm{O}$ to see $\mathrm{A}$, the relative velocity was $\mathrm{v}$. If the axial unit of time itself possessed relativity, its relative time changed t. From the reference system $\mathrm{O}$ to see $\mathrm{B}$, the relative velocity was $-\mathrm{v}$, and its relative time also changed t. In this regard, the two axial units of time of reference system $A$ and $B$ were equal and the same. Again from the reference system $B$ to see A, the relative velocity of reference system $A$ was $2 \mathrm{v}$, while the change in relative time was close to $2 t$, different from the situation observed from the reference system $O$. These two states were concurrent but contradictory. The only explanation was that the difference in value for these axial units of time $t=0$. With the same method, it could likely be deduced that the value of difference for the axial units of space $\mathrm{x}=0$. It was necessary to solve these contradictions and paradoxes with the conclusion that there was no relativity in time and space [2] [4].

In consistency, recently, it was demonstrated in updated experiments that the speed of neutrino was at least equal to that of light [5]. In consideration of that the neutrino possessed the mass, the result was unfavorable to the rationality of special relativity, while in further supported the non-relativity of ideal time and space. Besides, in the special theory of Einstein's relativity, the axiom the speed of light was identical for all inertial reference systems referred to the absolute ideal time and space since the electromagnetic fields would affect the light speed as manifested in light refraction, whereas in the special theory of Einstein's relativity the time and space was measured with physics, changing the concept in the theory.

\subsection{The Electromagnetic Mass, Multi-Hairy Structure and Orienting Force}

In the book, there were moreover introduced some novel electromagnetic structural forms and an important type of force, which were the keys to achieve the significant progressions, as followings:

1) The author Zi-Jian Cai and European people in television proposed that the mass of particle should possess the spatially localized electromagnetic structure from the fact that the $\gamma$ photon of high energy could transform in reciprocation with the gravitational mass [2] [6]. The mass electrical field and magnetic field of particle moved continuously and transformed reciprocally, but spatially this electromagnetic mass structure did not resemble that of photon which propagated away. Instead it returned to the vicinity around its original point of departure through its electromagnetic movement and transformation [2] [6]. Besides, the increase in mass during quark condensa- 
tion [7] [8] strongly supported the mass as spatially localized electromagnetic structure, with mass increment to be easily explained by electromagnetic conformational change [2] [6]. After Zi-Jian Cai led the sate-commune group to make this proposition in early October 2005, the activities in discussion and exchange of physics elicited the attention, comment and participation of people in the whole world with television as the common media.

2) The Sate-Commune Group India Member and athletes in video record of 2000 Olympic Opening Ceremony both proposed the electromagnetic multiple hairy structure [2] [9]. This structure and hybrid was beyond the consideration of the present quark model. In a photon when a changing electrical vector $E$ generated a changing magnetic vector $B$, immediately from such new and changing magnetic vector $B$ there generated the next changing electrical vector $\mathrm{E}$. At this time, the original electrical vector $\mathrm{E}$ had changed a little. Such process repeated continuously until the earliest changing electrical vector $\mathrm{E}$ vanished. In this way, there would generate the electromagnetic multi-hairy structure for the photon [2] [9]. In similarity, there would also generate the multi-hairy electrical fields for both positron and electron [2] [10]. It was easy to see that the positron and electron could hybrid like two gears.

3) A member of Sate-Commune Group with heavy Suzhou tone simply calling himself as Zhejiang proposed the electromagnetic orienting force [2] [11]. When several electromagnetic particles with positive and negative charges were close together, among their electrical fields there would occur the static electrical interactions with the same charge repelling and opposite charge attracting. The same charge repelling made the electrical fields increase in distance, decrease in interactive strength and decrease in interactive time. The opposite charge attracting made the electrical fields decrease in distance, increase in interactive strength and increase in interactive time. Such asymmetrical interactions made them generate a kind of net attractive force among the electromagnetic particles, called as the electromagnetic orienting force [2] [11].

\subsection{Electromagnetic Unification of Four Forces}

In the book, it was utilized the electromagnetic force to unify the strong force, weak force and gravitational force, unifying the four forces [1] [2]. In premise, the energy was present as the frequency of electromagnetic wave or frequency of light in physics, thereby all forces with energy should be unified with the electromagnetic force.

1) With regard to the strong force, it was suggested in the book that there formed the stable electromagnetic multi-hairy hybrid between the adjacent positive and negative quarks, while it was further divided the released energy brought about by the electromagnetic hybrid into two parts [2] [12]. One was the released energy of the electromagnetic multi-hairy hybrid itself. Another was the thermodynamic moving, vibrating and rotating energy of quark par- 
ticles and their internal electromagnetic structures, which was released by the orderliness and stability of electromagnetic multi-hairy hybrid [2] [12]. Both of the two energies were parts of the energy of strong interaction. The difference of $\pi$ meson and $\mu$ lepton in their nuclear reactivity supported the strong interaction as generated from the electromagnetic multi-hairy hybrid requiring matching on frequency [2] [12].

2) With regard to the weak process, it was suggested in the book that, as the electromagnetic multi-hairy hybrid of positive and negative quark was subject to the multiple destructions and effects such as the internal tension within atomic nucleus and so on, the hybrid was torn apart and there accordingly occurred the recombination and modification in structure of atomic nucleus. The electromagnetic multi-hairy hybrid torn-apart to single side formed weak pairing and binding with the external electrical field of opposite charge, getting stabilized temporarily [2] [13]. In the subsequent process of weak interactive decay, the external electrical field pairing and binding with the single side of hybrid turned to bind with another external electrical field of opposite charge in external environment, dropping away from its binding with the original single side of hybrid, and becoming the neutrino of oscillatory electrical structure of opposite charge [2] [13].This mechanism of weak interaction was able to explain the relatively small mass of neutrino and the reverse reaction of weak interaction [2] [13].

3) With regard to the gravitational force, it was suggested in the book that the electromagnetic mass waving entity of gravitational donor interacted in electromagnetic orientation with the electromagnetic waves mediating the gravity, making them turn in curve. While such orienting interaction fading away with the electromagnetic waves partially returning to the original status, the electromagnetic waves consumed energy and shifted red [2] [14]. Both of them manifested in sum as the phenomena of gravitational field [2] [14].The photon and electromagnetic wave curving and shifting red in gravitational field directly supported this explanation [2] [14]. Besides, the increment of mass during condensation of quarks [7] [8] could only be explained with the electromagnetic conformational change of gravitational entity, with higher mass frequency affected more in coordination in gravitational attraction, supporting this mechanism of gravitational force.

\section{The Electromagnetic Explanation of Classical Quantum Theory and Quantum Tunneling Effect}

The book was finished in writing in 2009, with its contents fixed since then. After that time, there have also been achieved new progressions. Herein, it is introduced the explanation of the classical quantum theory and quantum tunneling effect with the electromagnetic structures in the book [2].

Classical quantum theory has mostly been successful in description of the behavior of electron around the atomic nucleus. Notably, the quantum theory is 
capable of accurate calculating the relatively simple atoms as physical explanation of the periodic table [15]. Besides, the quantum tunneling effect manifests a particle tunnels through a barrier that it classically could not surmount [16], so as to refer to quantum theory necessarily.

It is necessary to analyze the photon electron interaction manifesting the quantum phenomena. It is obvious that any photon emitted by the electron would have the same electrical hairy strength as the electron [2]. With the repeated occurrence of photon-electron absorption and emission during the evolution of cosmos, all photons would acquire the same electrical hairy strength as that of electron. In this way, with the identical electrical hairy strength, the energy of all photons would be only relevant to their frequencies [2], which is exactly the phenomenon of Planck, the basis of quantum theory [2].

The quantum tunneling effect also deserves to be specially addressed [16]. For an electron to tunnel through a high barrier that it classically could not surmount, it is necessary to resort to the quantum wave property of electron. In the spatially localized electromagnetic mass structure, its electrical field and magnetic field move continuously and transform reciprocally, but spatially they return to the vicinity around their original point of departure [2] [6]. If there is deviation in returning back to the original point due to external effects, the electromagnetic electron would be able to manifest the quantum tunneling effect [2] [16]. This is also the strong evidence in support of the spatially localized electromagnetic structure of mass.

\section{Proposal of the Electromagnetic Structures of Important Particles from Decays}

\subsection{The Methods of Deducing the Electromagnetic Structures of Particles with Their Radiative and Electromagnetic Decay}

To determine the electromagnetic structure of particle, the radiative decays and electromagnetic decay of it can provide useful information on the structural constituents of the particle [3], as the followings: 1) From the mass frequency of particle, it is possible to determine the frequency of the particle and of the hybrid in the particle. 2) From the hadronic and weak interactive decays, it is possible to determine whether there is any electromagnetic hybrid in the particle, as well as the number of constituent particles in the combined particle. The bigger the particles generated in the decays, the more useful to clarify the structure of the combined particle. 3) Sometimes, it is possible that a constituent particle may have more than one hybrids in the combined particle. From the electromagnetic decay, it is possible to assist determination of the number of electromagnetic hybrids present in the particle.

In the book, with the weak interactive decay of charged $\pi$ meson to $\mu$ lepton and finally to positron and electron [2] [3] [13] [17] [18], it was deduced that the charged $\pi$ meson was made from the respective positron and electron with at least one electromagnetic hybrid in structure [2] [3] [13] [17], while with the 
electromagnetic decay of $\pi 0$ meson, it was deduced that the $\pi 0$ meson was just the dropped hybrid with average length [2] [3] [17].

The $\tau$ type neutrino is special in that it is the heaviest neutrino in all three types of neutrinos. From the electromagnetic neutrino structure [1] [2], the $\tau$ type neutrino should also be composed of the oscillatory electrical structure of opposite charge. On the other hand, the $\tau$ - lepton decays in many ways [19], with the most common three ways as: 1) $\tau_{-} \rightarrow \pi-\pi 0 \nu^{\tau}$; 2) $\tau_{-} \rightarrow \pi-v^{\tau}$; 3) $\tau_{-} \rightarrow$ $\pi-2 \pi 0 v^{\tau}$. With the variety in number of $\pi 0$ mesons generated, it is obvious that the $\tau$ - lepton contains a $\pi$ - meson with more than one hybrid, while the $\tau$ type neutrino possesses one electrical field able to adjust the angle of adjacent hybrid regions in the $\tau$ - lepton. Whereas, as $\tau$ - lepton may also decay in other minor ways generating more than one $\pi$ - mesons, it is likely that the $\tau$ - lepton may have several subtypes having not been purified yet.

The W/Z bosons possesses very big mass up to more than $80 \mathrm{GeV} / \mathrm{c}^{2}$ [20]. It has been suggested by the physicists that the big mass of $\mathrm{W} / \mathrm{Z}$ bosons corresponds to the short interactive distance of weak interaction [3] [20]. Because in the book the short interactive distance of weak interaction corresponds to the hybrid interval between the positive and negative electromagnetic entities [2] [13], it is obvious that the $\mathrm{W} / \mathrm{Z}$ bosons possess the wavelengths equivalent to that interval. Since usually the W/Z boson forms from proton collision, it is conceivable that the generated $\mathrm{W} / \mathrm{Z}$ boson of high frequency then turns to lock with the hybrid interval present around in quantity. Due to the impurity of the surrounding hybrids the $\mathrm{W} / \mathrm{Z}$ boson locks to, the W/Z boson decays in numerous ways [3] [20].

To deduce the structure of combined particle from its decays to the constituent particles, it is necessary to consider the conservation of energy (mass), charge and spin. It is necessary to point out that, in the book, the conservation of mass, energy, charge and spin was maintained in the decays of both $\pi$ mesons and $\mu$ leptons [3] [13] [17] [18], as well as in the electron/positron, neutrinos and photons [3] [13] [17]. If the decays from a bigger combined particle to them are also conserved in energy (mass), charge and spin, it would be certain that the deduced structure of the particle is as well conserved in mass, energy, charge and spin naturally.

Due to the huge number of particles, it is impossible to provide a full description of structures of all kinds of particles. In this regard, in this paper, it is only attempted to depict the structures of some particles most important in physics. Besides, due to the incompleteness in reliable data available for decays of some high-energy particles, it is also necessary to further improve and rectify the structures listed herein in future.

\subsection{Photon, Positron/Electron, $\pi$ Meson, $\mu$ Lepton, Neutrino, $\tau$ - Lepton, Up/Down Quark and Strange Quark}

In the book "Electromagnetic Unification of Four Forces" (ISBN978-3-659-76798-2), the world people worked out the electromagnetic structures for these important 
particles except the $\tau$ - lepton. The structures of all of these particles are listed in Table 1.

With the electromagnetic mechanism of weak process, it was further speculated in the book and afterwards that, if modifying the present quark theory and proposing that the positive and negative $\pi$ mesons themselves consisted of only one single quark, it was possible to construct the structural model for the positive and negative quark with the weak interactive decays of $\pi$ meson [2] [3] [17] [18], that was the positive and negative quark was just the charged $\pi$ meson made from the respective positron and electron with at least one electromagnetic hybrid [2] [3] [17] [18]. The defined charge and mass of them should also be revised accordingly, equivalent to those of corresponding $\pi$ mesons.

Such revision is supported in five aspects: 1) No free up/down quark has been found in experiments up to now [3] [21]. 2) The European people in television pointed out that the charge of positron/electron could not be further divided in experimental observations up to now, and accordingly the charge of respective charged $\pi$ mesons could neither be divided [3]. In this regard, it is most likely that one charged $\pi$ meson contains one charged quark without dividing the charge. 3) Quark condensation with increment in mass or frequency supports the explanation as formation of the electromagnetic hybrid [2] [3] [17]. 4) As

Table 1. The structures of photon, positron/electron, $\pi$ mesons, $\mu$ leptons, neutrinos, $\tau$ lepton, up/down quark and strange quark.

\begin{tabular}{|c|c|c|c|c|}
\hline Particles & Mass $\left(\mathrm{MeV} / \mathrm{c}^{2}\right)$ & Charge & Decays & Structure \\
\hline Photon [2] [9] & 0 & 0 & Stable & \\
\hline Electron [2] [10] & 0.511 & $-\mathrm{e}$ & Stable & 䊉 \\
\hline Positron [2] [10] & 0.511 & $+\mathrm{e}$ & Stable & 唯 \\
\hline$\mu \pm$ leptons & 106.6 & $\pm \mathrm{e}$ & $\mu \pm \rightarrow \mathrm{e} \pm v^{\mathrm{e}} v^{\mu}$ & \\
\hline$\pi \pm$ mesons & 139.6 & $\pm \mathrm{e}$ & $\pi \pm \rightarrow \mu \pm v^{\mu}[2][17][18]$ & \\
\hline$\pi 0$ mesons & 135.0 & 0 & $\pi 0 \rightarrow \gamma[2][17]$ & \\
\hline $\begin{array}{l}\text { Neutrinos } \\
{[2][13][17]}\end{array}$ & Sum 0.00032 & 0 & Stable & \\
\hline$\tau$ - leptons & 1777 & $-\mathrm{e}$ & $\begin{array}{c}\tau-\rightarrow \text { (a) } \pi-\pi 0 v^{\tau} \text {; } \\
\text { (b) } \pi-v^{\tau} \text {; (c) } \pi-2 \pi 0 v^{\tau} \text {; } \\
\text { et al. [3] [18] }\end{array}$ & \\
\hline Up quark & 139.6 & $+\mathrm{e}$ & $\begin{array}{c}\pi+\rightarrow \mu+v^{\mu} \\
{[2][3][17][18]}\end{array}$ & \\
\hline Down quark & 139.6 & $-\mathrm{e}$ & $\begin{array}{c}\pi-\rightarrow \mu-v^{\mu} \\
{[2][3][17][18]}\end{array}$ & \\
\hline Strange quark & $95 \pm 5$ & 0 & $\pi 0 \rightarrow \gamma[2][3][17]$ & $\bar{E}$ \\
\hline
\end{tabular}

Notes: (a) Mass of neutrinos is the average summed mass of all 3 types of neutrinos. (b) $\tau$ - leptons may be unpurified mixtures of similar characteristics, with the charge rectified to that of $\pi$ meson. (c)The mass and charge of up and down quark are both rectified equivalent to $\pi \pm$ mesons. 
there are only one kind of photon, without differentiation in photons emitted by electron and by nuclear quark [2] [3], it supports the speculation that the positive and negative quark were just the respective positron and electron with at least one hybrid [2] [3] [17]. 5) The present quark theory assuming the charged $\pi$ mesons composed of two quarks is difficult to account for the difference of $\pi$ meson and $\mu$ lepton in their nuclear reactivity [2] [12].

Witnessed by the author, it was even speculated by the European people in television that the color of up/down quark was related to the number of electromagnetic hybrids within one quark, which needs further investigation.

As the decays of the particles containing the strange quark often involve the generation of $\pi 0$ particle which can directly decay into $\gamma$ photons electromagnetically, it is very likely that the decays of these particles through strange quark just represent the deletion of the hybrid region. The mass of strange quark at around $100 \mathrm{MeV} / \mathrm{c}^{2}$ [24] supports this proposal.

\subsection{Proton, Neutron, and the $\Lambda, \Sigma, \Xi$ Baryons}

From the products of decays, it is possible to construct the structure of combined particles. Herein, it is introduced the structures of the important combined particles related to proton, including the proton, neutron, and the $\Lambda, \Sigma, \Xi$ baryons, as listed in Table 2 . Whereas, the $\Delta$ baryons are too short in life times to be listed herein.

Table 2. The structures of proton, neutron, and the $\Lambda, \Sigma, \Xi$ baryons.

\begin{tabular}{|c|c|c|c|c|}
\hline Particles & Mass $\left(\mathrm{MeV} / \mathrm{c}^{2}\right)$ & Charge & Decays & Structure \\
\hline $\begin{array}{l}\text { Proton } \\
{[2][3][17]}\end{array}$ & 938.3 & $+\mathrm{e}$ & Stable & \\
\hline Neutron [22] & 939.6 & 0 & $\begin{array}{c}\mathrm{n} 0 \rightarrow(\mathrm{a}) \mathrm{p}+\mathrm{e}-v^{\mathrm{e}} ;(\mathrm{b}) \mathrm{p}+\mathrm{e}-v^{\mathrm{e}} \gamma \\
{[3][22]}\end{array}$ & \\
\hline$\Lambda 0$ baryon & 1115.6 & 0 & $\Lambda 0 \rightarrow$ (a) $p+\pi-;$ (b) n $\pi 0[3][23]$ & \\
\hline$\Sigma+$ baryon & 1189.4 & $+\mathrm{e}$ & $\begin{array}{c}\Sigma+\rightarrow(\mathrm{a}) \mathrm{n} \pi+; \text { (b) } \mathrm{p}+\pi 0[3] \\
{[24]}\end{array}$ & \\
\hline$\Sigma$ - baryon & 1197.3 & $-e$ & $\Sigma-\rightarrow \mathrm{n} \pi-[3][24]$ & \\
\hline 20 baryon & 1192.5 & 0 & $\Sigma 0 \rightarrow \Lambda 0 \gamma[3][25]$ & \\
\hline$\Xi$-baryon & 1321.2 & $-\mathrm{e}$ & $\Xi-\rightarrow \Lambda 0 \pi-[3][26]$ & \\
\hline$\Xi 0$ baryon & 1314.7 & 0 & $\Xi 0 \rightarrow \Lambda 0 \pi 0[3][26]$ & \\
\hline
\end{tabular}

Notes: (a) $€ \oplus$ is the multiple hairy hybrid of positive and negative quarks by flat plane; (b) $(-\oplus$ is the intimate electrical interaction between the electron and positive quark. 


\subsection{The $\mathrm{K}, \eta$ Baryons, and the Charm and Bottom Quarks}

There are other types of baryons very unstable, including the $\mathrm{K}, \eta$ baryons and so on, as well as the charm and bottom quarks. They decay quickly through kaon-related structure in various modes, including $2 \pi / 3 \pi$ distinct modes often containing $\pi 0$ products. They obviously contain the mixture of unpurified colliding subtypes of baryons of similar characteristics [3]. The deduced structures of all of these particles are listed in Table 3.

\subsection{The W/Z/Higgs Bosons and the Top Quark}

The W/Z/Higgs Bosons and the Top Quark are all big in mass of more than $80 \mathrm{GeV} / \mathrm{c}^{2}$ [3] [20] [33] [34] [35]. It has been suggested by the physicists that the big mass of W/Z bosons corresponds to the short interactive distance of weak interaction [20], so that it is obvious that the $\mathrm{W} / \mathrm{Z}$ bosons have the wavelengths equivalent to the hybrid interval between the positive and negative electromagnetic entities [3] [13] [17]. The structures of all of these particles are listed in Table 4.

\section{Discussions}

In this article, it is reviewed the important contents of the milestone book "Electromagnetic Unification of Four Forces" (ISBN978-3-659-76798-2) [1] [2],

Table 3. The structures of the $\mathrm{K}, \eta$ baryons, and the charm and bottom quarks.

\begin{tabular}{|c|c|c|c|c|}
\hline Particles & $\operatorname{Mass}\left(\mathrm{MeV} / \mathrm{c}^{2}\right)$ & Charge & Decays & Structure \\
\hline $\mathrm{K} \pm$ baryons & 493.8 & $\pm \mathrm{e}$ & $\begin{array}{c}\mathrm{K} \pm \rightarrow \pi \pm \pi 0[3][27] \\
{[28]}\end{array}$ & \\
\hline K0 baryon & 497.8 & 0 & $\begin{array}{l}\mathrm{K} 0 \rightarrow \text { (a) } 2 \pi \text {; (b) } 3 \pi ; \\
\quad \text { et al. }[3] \text { [27] }\end{array}$ & \\
\hline$\eta$ baryon & 548.8 & 0 & $\begin{array}{c}H \rightarrow \text { (a) } 3 \pi 0 ; \\
\text { (b) } \pi 0 \pi+\pi-; \\
\text { et al. [3] [29] [30] }\end{array}$ & \\
\hline Charm quark \pm & 1275 & $\pm \mathrm{e}$ & $\begin{array}{c}\mathrm{D} 0 \rightarrow(\mathrm{a}) \mathrm{K}-\pi 0 \pi+;(\mathrm{b}) \\
\mathrm{K}-\pi+\pi+\pi-; \text { et al. }[3] \\
{[31]}\end{array}$ & \\
\hline Bottom quark \pm & 4180 & $\pm \mathrm{e}$ & $\mathrm{b} \pm \rightarrow \mathrm{c} \pm \mathrm{lv}$; et al. [3] [32] & \\
\hline
\end{tabular}

Notes: (a) Most of these particles represent unpurified colliding mixtures of similar characteristics. (b) The charges of charm quark and bottom quark are both rectified equivalent to $\pi \pm$ mesons. 
Table 4. The structures of the W/Z/Higgs bosons and the top quark.

\begin{tabular}{|c|c|c|c|c|}
\hline Particles & $\begin{array}{c}\text { Mass } \\
\left(\mathrm{MeV} / \mathrm{c}^{2}\right)\end{array}$ & Charge & Decays & Structure \\
\hline $\mathrm{W} \pm$ bosons & 80385 & $\pm \mathrm{e}$ & $\begin{aligned} \mathrm{W} \pm \rightarrow & \text { (a) } \mathrm{c}+\mathrm{b}-; \text { (b) b+b-; } \\
& \text { et al. [3] [33] }\end{aligned}$ & E Hybrid interval \\
\hline $\mathrm{Z}$ boson & 91188 & 0 & $\begin{array}{c}\mathrm{Z} \rightarrow(\mathrm{a}) \mathrm{c}+\mathrm{b}-;(\mathrm{b}) \mathrm{b}+\mathrm{b}-; \\
\text { et al. }[3][20]\end{array}$ & 其 Hybridinerval \\
\hline Higgs boson & 125090 & 0 & $\begin{array}{c}\text { Higgs } \rightarrow \text { (a) W+W-; (b) ZZ; } \\
\text { et al. [3] [34] }\end{array}$ & 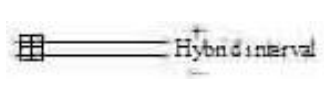 \\
\hline Top quarks \pm & 173210 & $\pm \mathrm{e}$ & $\begin{array}{c}\mathrm{t} \pm \rightarrow \text { (a) } \mathrm{W} \pm \mathrm{c} \pm ;(\mathrm{b}) \mathrm{W} \pm \mathrm{b} \pm \\
\text { et al. }\end{array}$ & 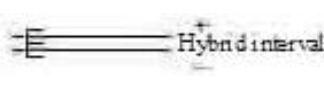 \\
\hline
\end{tabular}

Notes: (a) These structural drawings only show the high frequency piece locking to the hybrid interval, with the rest parts unshown. (b) Each particle represents unpurified colliding mixture of similar characteristics. (c) The charges of top quarks are rectified equivalent to $\pi \pm$ mesons.

introducing the non-relativity of ideal time and space, the spatially localized electromagnetic mass structure, the electromagnetic multi-hairy structure and the electromagnetic orienting force, and demonstrating how to unify the four forces with the electromagnetic force. The strong force resulted from the stable electromagnetic multi-hairy hybrid between the adjacent positive and negative quarks, releasing the electromagnetic multi-hairy hybrid energy itself, and releasing the thermodynamic moving, vibrating and rotating energy of quark particles by the orderliness and stability of electromagnetic multi-hairy hybrid, both of which contributed to the strong force. The weak process resulted from the electromagnetic hybrid torn apart to form weak pairing and binding with the external electrical field of opposite charge, getting stabilized temporarily. Then, the external electrical field pairing and binding with the single side of hybrid turned to bind with another external electrical field of opposite charge in external environment, dropping away from its binding with the original single side of hybrid, and becoming the neutrino of oscillatory electrical structure. The gravitational force resulted from the electromagnetic mass waving entity interacting in electromagnetic orientation with the electromagnetic waves mediating the gravity, making them turn in curve and shift to red [1] [2].

In this review, it is in further collected some important progressions after the book, in order to acquire the ability to comprehensively explain all experimental phenomena and structures of important particles in physics.

When dealing with the quantum theory with electromagnetic electron wave, it resorts to a reasonable constraint, that is the photon emitted by electron should have the identical electrical hairy strength as that of electron [2]. Accordingly, all photons from electron should have the same electrical hairy strength, so that the energy of photons is only relevant to the frequencies of photons [2], manifesting the phenomenon of Planck as the basis of quantum theory [2].

Afterwards, it is certainly important to propose the electromagnetic structures of important particles in physics. All the proposed structures are listed in the 
above several Tables and convenient for readers to refer and cite. From these many particle structures, ranging from the most basic particles such as electron, neutrino to the huge colliding particles such as Higgs boson, it is possible to classify their structures into four types.

1) The simple stable structure: Many basic and common particles have this type of structure, which are different from each other. Among them, there are the electromagnetic multiple hairy structure of photon, the multiple electrical hairy structure of positron and electron, the oscillatory electrical structure of neutrino, the charged $\pi$ meson and up/down quark as the respective positron/electron with at least one electromagnetic hybrid of higher frequency.

2) The combined structure based on linear proton and hexagonal neutron: Many combined particles possess this structure with proton of five quarks in linear structure or neutron with hexagonal plane. Among them, there are the proton, the neutron, the $\Lambda 0$ baryon, the $\Sigma$ baryons, and the $\Xi$ baryons. The $\Delta$ baryons are too short in life times to be listed.

3) The combined structure based on kaons: Many unstable combined particles possess the structure with $\mathrm{K}$ baryons from colliding pieces of various sizes and characteristics as the major constituents. Among them, there are the kaons, the $\eta$ baryons, the charm quarks, and the bottom quarks.

It is necessary to note that some particles from proton collision, such as $\Omega$ baryon, can decay into both proton-type baryon and K-type baryon, as $\Omega-\rightarrow$ $\Lambda \mathrm{K}-\rightarrow(\mathrm{p} \pi-) \mathrm{K}-[36]$. Their structures can similarly be worked out from the decay products as others.

4) The combined structure of high frequency containing W/Z bosons: Some unstable combined particles from high-energy collision possess the very high frequency as that of W/Z bosons, whose wavelength can match to the hybrid interval of two particles of opposite charge. Among them, there are the W/Z bosons, the Higgs boson, and the top quarks.

\section{Conclusions}

This article reviews the important contents of the milestone book "Electromagnetic Unification of Four Forces" (ISBN978-3-659-76798-2), including the non-relativity of ideal time and space, the spatially localized electromagnetic mass structure, the electromagnetic multi-hairy structure and the electromagnetic orienting force. It also reviewed the electromagnetic unification of four forces in the book. The strong force formed from energy release of the electromagnetic multi-hairy hybrid energy itself, and energy release of the thermodynamic moving, vibrating and rotating energy of quark particles by the orderliness and stability of electromagnetic multi-hairy hybrid. The weak process manifested the electromagnetic hybrid torn apart to form weak pairing and binding with the external electrical field of opposite charge. Then, the pairing and binding external electrical field turned to bind with another external electrical field of opposite charge in external environment, dropping away from its binding with 
the original single side of hybrid, and becoming the neutrino of oscillatory electrical structure. The gravitational force was the electromagnetic mass waving entity interacting in electromagnetic orientation with the electromagnetic waves, making them turn in curve and shift to red.

It also collects some important progressions after the book. It is suggested that the electromagnetic mass structure can explain the quantum theory of Planck because all photons emitted from electron have identical electrical hairy strength as electron with frequency to differentiate energy only.

It further proposes the electromagnetic structures of particles important in physics, including the positron/electron, neutrino, charged $\pi$ meson, up quark, down quark, strange quark, and $\tau$ - leptons; the combined baryons based on linear proton and hexagonal neutron such as the proton, neutron, $\Lambda 0$ baryon, $\Sigma$ baryons, and $\Xi$ baryons; the combined structures based on kaons from colliding pieces of various sizes and characteristics such as the kaons, $\eta$ baryons, charm quarks, and bottom quarks; the combined structures from colliding pieces of high frequency containing W/Z bosons, such as the W/Z bosons, Higgs boson, and top quarks. All of these electromagnetic structures are constructed from their decay products. All of these structural results are listed into tables convenient for physicists to refer and cite.

\section{Acknowledgements}

It is herein acknowledged that Mingxun Cai paid the Open Access publication fee of this paper.

\section{Funding}

The author declares no financial support for this work.

\section{Conflict of Interest Statement}

The author declares no conflict of interest for this work.

\section{References}

[1] Cai, Z.-J. (2015) Electromagnetic Unification of Four Forces. Lambert Academic Publishing, Saarbrücken.

[2] Cai, Z.-J. (2016) Review of Milestone Book: Electromagnetic Unification of Four Forces. Research \& Reviews. Journal of Pure and Applied Physics, 4, 20-27.

[3] Cai, Z.-J. (2016) Proposal of the Electromagnetic Structures of the Most Important Particles from Decays. Research \& Reviews. Journal of Pure and Applied Physics, 4 , 9-19.

[4] Cai, Z.-J., Sports-People 2000, O.O.C., Sports-People 2004, O.O.C., Beijing Member, S.C.G., Jiang, K.T.V. and Hongkong People, T.V. (2015) Disproving the Relativity of Ideal Time and Space. In: Cai, Z.-J., Ed., Electromagnetic Unification of Four Forces, Lambert Academic Publishing, Saarbrücken, 11-16.

[5] Antonello, M., Aprili, P., Baiboussinov, B., Baldo Ceolin, M., Benetti, P., Calligarich, E., Canci, N., et al. (ICARUS Callaboration) (2012) Measurement of the Neutrino 
Velocity with the ICARUS Detector at the CNGS Beam. Physics Letters B, 713, 17-22. https://doi.org/10.1016/j.physletb.2012.05.033

[6] Cai, Z.-J., Beijing Member, S.C.G., European People, T.V. and Southern American People, T.V. (2015) Explaining the Mass with the Spatially Localized Electromagnetic Structure. In: Cai, Z.-J., Ed., Electromagnetic Unification of Four Forces, Lambert Academic Publishing, Saarbrücken, 34-37.

[7] Crewther, R.J. (1986) Testing the Mode of Quark Condensation. Physics Letters B, 176, 172-178. https://doi.org/10.1016/0370-2693(86)90945-7

[8] Dai, Y.B., Ding, Y.B., Huang, C.S. and Wang, C.L. (1992) Top Quark Mass in Top Quark Condensation Model. Physics Letters B, 285, 245-250.

https://doi.org/10.1016/0370-2693(92)91460-Q

[9] India Member, S.C.G., Cai, Z.-J., Sports-People 2000, O.O.C., Sports-People 2004, O.O.C., Zhejiang Members, S.C.G., Beijing Member, S.C.G. and Chinese People, T.V. (2015) The Electromagnetic Multi-Hairy Structure and the Photon. In: Cai, Z.-J., Ed., Electromagnetic Unification of Four Forces, Lambert Academic Publishing, Saarbrücken, 43-48.

[10] India Member, S.C.G., Cai, Z.-J., Sports-People 2004, O.O.C., Shanghai People, T.V., European People, T.V. and Southern American People, T.V. (2015) The Electromagnetic Multi-Hairy Structure and the Particle of Charge. In: Cai, Z.-J., Ed., Electromagnetic Unification of Four Forces, Lambert Academic Publishing, Saarbrücken, 49-53.

[11] Zhejiang Members, S.C.G. and Shanghai Member, S.C.G. (2015) The Electromagnetic Orienting Force. In: Cai, Z.-J., Ed., Electromagnetic Unification of Four Forces, Lambert Academic Publishing, Saarbrücken, 38-42.

[12] UN Staffs, T.V., India Members, S.C.G., US Members, S.C.G., US Congress Senator, T.V., Cai, Z.-J., Zhejiang Members, S.C.G., Korean People, T.V. and European People, T.V. (2015) Explaining the Strong Interaction with the Electromagnetic Multi-Hairy Hybrid. In: Cai, Z.-J., Ed., Electromagnetic Unification of Four Forces, Lambert Academic Publishing, Saarbrücken, 59-66.

[13] Taiwan Member, S.C.G., Beijing Members, S.C.G., European People, T.V., Shenzhen People, T.V., Guangdong Member, S.C.G., Zhejiang Members, S.C.G., Cai, Z.-J., India Member, S.C.G., Chinese People, T.V. and Nanjing People, T.V. (2015) Explaining the Weak Interaction with the Electromagnetic Interaction. In: Cai, Z.-J., Ed., Electromagnetic Unification of Four Forces, Lambert Academic Publishing, Saarbrücken, 76-83.

[14] Shanghai Member, S.C.G., US People, T.V., Nanjing People, T.V., US Members, S.C.G., Southern American People, T.V., Zhejiang Members, S.C.G. and Beijing People, T.V. (2015) Explaining the Gravitational Field of General Relativity with the Electromagnetic Wave in Curve and Red Shift. In: Cai, Z.-J., Ed., Electromagnetic Unification of Four Forces, Lambert Academic Publishing, Saarbrücken, 67-72.

[15] Ostrovsky, V.N. (2003) Physical Explanation of the Periodic Table. Annals of the New York Academy of Sciences, 988, 182-192. https://doi.org/10.1111/j.1749-6632.2003.tb06097.x

[16] Ionescu, A.M. and Riel, H. (2011) Tunnel Field-Effect Transistors as Energy-Efficient Electronic Switches. Nature, 479, 329-337. https://doi.org/10.1038/nature10679

[17] Beijing People, T.V., US People, T.V., Cai, Z.-J., European People, T.V., Shanghai People, T.V., Zhejiang Members, S.C.G., Beijing Member, S.C.G., India Member, 
S.C.G. and Artists/Audience, S.S.P. (2015) Two Possibilities for the Relationship between the Quark and $\pi$ Meson. In: Cai, Z.-J., Ed., Electromagnetic Unification of Four Forces, Lambert Academic Publishing, Saarbrücken, 84-92.

[18] Castagnoli, C. and Muchnik, M. (1958) Experimental Results on the Radiative $\pi$ - $\mu$ Decay. Physical Review, 112, 1779-1784. https://doi.org/10.1103/PhysRev.112.1779

[19] Gan, K.K. (2004) New Results on $\tau$ Lepton. The European Physical Journal C, 33, s647-s649. https://doi.org/10.1140/epjcd/s2004-03-1612-4

[20] Wikipedia, W and Z Bosons. https://en.wikipedia.org/wiki/W_and_Z_bosons

[21] Lyons, L. (1981) Current Status of Quark Search Experiments. Progress in Particle and Nuclear Physics, 7, 157-167. https://doi.org/10.1016/0146-6410(81)90014-4

[22] Wikipedia, Neutron. https://en.wikipedia.org/wiki/Neutron

[23] Dalitz, R.H. and Liu, L. (1959) Pionic Decay Modes of Light $\Lambda$ Hypernuclei. Physical Review, 116, 1312-1321. https://doi.org/10.1103/PhysRev.116.1312

[24] Barshay, S. (1962) Theory of the Pionic Decay of Sigma Hyperons in the Bound State Model. Annals of Physics, 18, 1-11. https://doi.org/10.1016/0003-4916(62)90055-6

[25] Dolgov, A.D. and Zakharov, V.I. (1974) Decay $\Sigma+/--->\Lambda$ ev and 20 Life Time. Physics Letters B, 48, 43-44. https://doi.org/10.1016/0370-2693(74)90453-5

[26] Yeh, N., Gaigalas, A., Smith, W.D. and Habibi, M. (1974) Observation of Rare Decay Modes of the $\Xi$ Hyperons. Physical Review D, 10, 3545. https://doi.org/10.1103/PhysRevD.10.3545

[27] Choudhury, S.R. and Scadron, M.D. (1995) Chiral Calculations of the $\mathrm{K} \rightarrow 2 \pi$ and $\mathrm{K} \rightarrow 3 \pi$ Weak Decays. IINuovoCimento A, 108, 289-298. https://doi.org/10.1007/BF02787056

[28] Ambrosino, F., Antonelli, A., Antonelli, M., Archilli, F., et al. (2008) Measurement of the Branching Ratio of the $\mathrm{K}(+) \rightarrow \mathrm{pi}(+) \mathrm{pi}(0)$ (gamma) Decay with the KLOE Detector. Physics Letters B, 666, 305-310. https://doi.org/10.1016/j.physletb.2008.07.070

[29] Aaron, R. and Goldberg, H. (1980) $\eta$ Decay and the Quark Structure of the $\varepsilon$. Physical Review Letters, 45, 1752. https://doi.org/10.1103/PhysRevLett.45.1752

[30] Frenkel, A., Vesztergombi, G. and Marx, G. (1970) C-Violation in $\eta$-Decay? Nuclear Physics B, 15, 429-441. https://doi.org/10.1016/0550-3213(70)90072-6

[31] Libbya, J., Malde, S., Powell, A., Wilkinson, G., Asner, D.M., Bonvicini, G., Briere, R.A., Gershon, T., Naik, P., Pedlar, T.K., Rademacker, J., Ricciardi, S. and Thomas, C. (2014) New Determination of the D $0 \rightarrow \mathrm{K}^{-} \pi^{+} \pi^{0}$ and $\mathrm{D} 0 \rightarrow \mathrm{K}^{-} \pi^{+} \pi^{+} \pi^{-}$Coherence Factors and Average Strong-Phase Differences. Physics Letters B, 731,197-203. https://doi.org/10.1016/j.physletb.2014.02.032

[32] Pak, A., Blokland, I. and Czarnecki, A. (2006) Two-Loop QCD Corrections to Semileptonic b-Quark Decays near Maximum Recoil. Physical Review D, 73, Article ID: 114009. https://doi.org/10.1103/PhysRevD.73.114009

[33] Liao, Q.L., Wu, X.G., Jiang, J., Yang, Z., Fang, Z.Y. and Zhang, J.W. (2012) Excited Heavy Quarkonium Production at the LHC through W Boson Decays. Physical Review D, 86, Article ID: 014031. https://doi.org/10.1103/PhysRevD.86.014031

[34] Bredenstein, A., Denner, A., Dittmaier, S. and Weber, M.M. (2006) Precise Predictions for the Higgs-Boson Decay $\mathrm{H} \rightarrow \mathrm{WW} / \mathrm{ZZ} \rightarrow 4$ Leptons. Physical Review D, 74, Article ID: 013004. https://doi.org/10.1103/PhysRevD.74.013004

[35] Abazov, V.M., et al. (D0 Collaboration) (2005) Search for Right-Handed W Bosons 
in Top Quark Decay. Physical Review D, 72, Article ID: 011104(R). https://doi.org/10.1103/PhysRevD.72.011104

[36] Aaltonen, T., et al. (CDF Collaboration) (2009) Observation of the $\Omega$-b Baryon and Measurement of the Properties of the $\Xi$-b and $\Omega$-b Baryons. Physical Review $D, 80$, Article ID: 072003. https://doi.org/10.1103/PhysRevD.80.072003 